\title{
Optimization of Neutron Detection Module based on Li-Glass scintillator and an array of SIPMs
}

\author{
Udi Wengrowicz*, Alon Osovizky, Aaron Ocherashvili, Dimitry Ginzburg, Yair Ifergan, Eli Volasky, \\ Yagil Kadmon, Avi Raveh, Itzhak Orion \\ * NRCN and Ben-Gurion University of the Negev \\ udiwe@post.bgu.ac.il
}

\section{INTRODUCTION}

$\mathrm{N}$ EUTRONS do not interact directly with electrons in matter; therefore, the mechanisms for detecting neutrons are based on indirect methods through nuclear reactions in converter materials. Lithium-6 $\left({ }^{6} \mathrm{Li}\right)$ is considered among one of the most effective converters for thermal neutrons. In this work we investigated the performances of ${ }^{6} \mathrm{Li}$-Glass scintillators coupled to state-ofthe-art light sensors, arrays of Silicon Photomultipliers (SiPM) are presented. According to set-up models described here, PMT light sensors, used for decades, can be effectively replaced with SiPM sensors while maintaining and even improving the detection efficiency and reducing dimensions, weight and energy consumption.

Neutron detection is a key component of applications in national and homeland security, industry, and science. In the last two decades, the need for highly sensitivity neutron detectors for Homeland Security (HLS) applications has increased significantly. Therefore, novel technologies and devices are constantly being investigated. Specifically, neutron detectors are important because of their ability to detect small amounts of Special Nuclear Materials (SNMs) that could be used in hostile actions. SNMs are radioactive, and the radiation types that might be detectable a few meters from a hidden source are gamma rays and neutrons. Gamma-ray emissions are plentiful and span a wide range of energies, but they are difficult to distinguish from background gamma radiation and can be easily confused with medical, industrial or Naturally Occurring Radioactive Material sources. In contrast, neutron

Index Terms - Gamma rays, MCNP, Neutron detection, Numerical Simulations, Radiation Detectors, Scintillation counters.

U. Wengrowicz is with the NRCN (1), P.O. Box 9001, Beer-Sheva, 84190 , Israel. Now he is also with the Nuclear Eng. unit, Ben-Gurion University of the Negev(3), Beer-Sheva, 84105, Israel (e-mail: udiwe@post.bgu.ac.il).

A. Ocherashvili, Y. Ifergan and Y. Kadmon are with the NRCN (1), P.O. Box 9001, 84190, Beer-Sheva,

A. Osovizky, E. Volasky, D. Ginzburg and A. Raveh are with Rotem Industries Ltd.(2), Israel.

I. Orion is with the Nuclear Eng. unit, Ben-Gurion University of the Negev(3), Beer-Sheva, 84105, Israel (e-mail: iorion@bgu.ac.il). detection possesses two intrinsic benefits, a unique signature and long attenuation lengths, also in the gammaabsorbing shield. Therefore, the mere presence of neutrons above background levels is an excellent indicator of SNMs [1].

The essential requirements for all types of neutron detectors are high intrinsic efficiency, large sensitive area at a reasonable cost and effective rejection of gamma rays. For HLS applications, slow neutron detectors are generally used. In these instruments, the detection of neutrons is based on moderating (slowing down) their kinetic energy and then absorbing the neutrons with a target material called a converter. Desirable converter characteristics include high neutron absorption cross-section, minimal gamma-ray interactions and high Q-value. In reactions where all or most of the Q-value energy is delivered to heavy charged particles, there are several pulse processing methods that allow the discrimination of low-amplitude pulses related to gamma interactions and reliable measurement of pulses related to neutron absorption reactions.

Gas proportional detectors based on ${ }^{3} \mathrm{He}$ isotope possess most of the required qualities for slow neutron detection. Therefore, this technology has been for decades the traditional "gold standard" for neutron detection. By far, the most common source of ${ }^{3} \mathrm{He}$ is the decay of tritium. Starting in about 2001, consumption has risen rapidly, greatly surpassing production. Due to the extreme use of this isotope, the global reserves have been practically exhausted. The shortage of ${ }^{3} \mathrm{He}$ has triggered the search for an effective neutron detection alternative[2]. ${ }^{6} \mathrm{Li}$ is considered among one of the most effective converters for thermal neutrons, having cross-section of 940 barn via the reaction ${ }^{6} \mathrm{Li}(\mathrm{n}, \alpha){ }^{3} \mathrm{H}+4.78 \mathrm{MeV}$, a capture reaction in which all the Q-value is released to charged particles. Hence, in this work, we investigated the use of ${ }^{6} \mathrm{Li}$-Glass scintillators coupled to arrays of SiPMs for personal and mobile neutron detectors. Scintillation counters based on Li-Glass scintillators coupled to PMT are suitable for harsh environments. The newest developments based on SiPMs enable high gain and the low-light detection capabilities of the PMT while offering the benefits of a solid-state sensor: low-voltage operation, insensitivity to magnetic fields, reduced dimensions, mechanical robustness and excellent uniformity of response[3]. These traits make the SiPM of great interest for applications in portable radiation 
detection instrumentation based on scintillation material. According to existing commercial technologies, the effective detection area of a single SiPM is less than 0.4 $\mathrm{cm}^{2}$. Therefore, when working with large scintillators, the collection efficiency of the crystal output light decreases, which affects the sensitivity of the measured signal. To overcome these, in this study, we investigated the use of arrays of SiPMs with a combined sensing area that fits the scintillation dimensions. Models of neutron sensors consisting of Li-Glass Scintillators coupled to arrays of SiPMs are presented in this work. These models enable effective gamma-ray rejection and a neutron efficiency that comply and even exceed the requirements of the IEC 62534 standards[4] for portable neutron search detectors for homeland security applications.

\section{METHODS}

The detector developed in this work consists of a $50 \mathrm{~mm}$ $\times 50 \mathrm{~mm} \mathrm{Li-Glass}$ scintillator of different thicknesses attached to an array of SiPMs. Neutron detection performances and gamma rejection evaluation of $1 \mathrm{~mm}$ and $2 \mathrm{~mm}$ thick scintillators, type GS20 ${ }^{\circledR}$ made by Scintacor Ltd.[5], are presented. These scintillators consist of a Ce-activated lithium aluminosilicate glass with a lithium content of $6.6 \%$, where the lithium is isotopically enriched to $95 \%{ }^{6} \mathrm{Li}$. Since only about 6000 photons are emitted per absorbed neutron, a photo-sensor with an effective light detection is required. To improve the scintillator light collection, we used arrays of the newest series of SiPM, type Sensl $\AA$ ARRAYJ-60035-64P $\AA$. This device, that consists of an array of $8 \times 8$ SiPMs, $6 \mathrm{~mm} \times 6$ $\mathrm{mm}$ each, has an overall sensitivity equal to or even higher than the sensitivity of most of commercial PMTs. The array is assembled in a $51 \mathrm{~mm} \times 51 \mathrm{~mm}$ board. That way, one face of this board totally covers one face of the scintillator; the other side of this board includes two 80way connectors. Via a mating board-to-board connector, bias voltage is applied to all SiPMs, but the output of each SiPM can be measured separately. Since we need to measure the response of the scintillator as a whole unit all the $64 \mathrm{SiPM}$ output signals were connected together to an electronic card that includes amplifier, voltage comparator and neutron threshold set-up. The module also includes a polyethylene moderator in front of the scintillator and a reflector in the back. The developed neutron sensor probe, presented in Figure 1, is more rigid than similar sensors coupled with PMTs, reducing the dimensions and power consumption and preserving and even improving the physical properties of the detector.

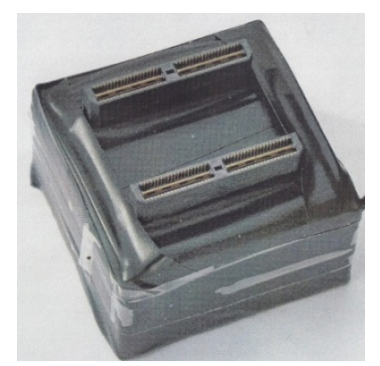

Figure 1: Neutron detection probe assembled in a rugged configuration.

\section{SIMULATIONS AND MEASUREMENTS}

For the detection and measurement of neutron sources, the scintillator composition, its thickness, the moderator materials and dimensions play an important role in the sensitivity of the instrument. By MCNP numerical simulations [6], we evaluated the sensitivity of the scintillators used in this work, Li-Glass type GS20®. The simulated detector consists of $5 \times 5 \mathrm{~cm}^{2}$ Li-Glass scintillator and $3 \mathrm{~mm}$ thick electronic cards including SiPM array and connection card. The simulations define also a reflector, $2 \mathrm{~cm}$ thick on each side of the scintillator and $4 \mathrm{~cm}$ thick behind the scintillator and the electronic cards. For a ${ }^{252} \mathrm{Cf}$ neutron source, the sensitivity of $1 \mathrm{~mm}$ and $2 \mathrm{~mm}$ thick scintillators was evaluated for different moderator thicknesses. The highest efficiencies were obtained with a $4 \mathrm{~cm}$ moderator. For thicker moderators, efficiency is reduced due to self-absorption.

The performances required for the hand-held neutron search detectors were defined by the IEC 62534 standard in the previous decade. Test methods defined by the standard require the detection within a period of 2 seconds of a $20000 \mathrm{n} / \mathrm{s}{ }^{252} \mathrm{Cf}$ source placed $125 \mathrm{~cm}$ from the instrument. The specified maximum dimensions of the instrument are $300 \mathrm{~mm} \times 200 \mathrm{~mm} \times 150 \mathrm{~mm}$, with a maximum allowed weight of $6 \mathrm{~kg}$. To meet IEC 62534 required sensitivity, the instrument efficiency must be at least $25 \mathrm{cps} / \mathrm{nv}$, and the average background rate must be less than $0.05 \mathrm{cps}$. Until the previous decade, it was possible to meet these requirements with a detector based on ${ }^{3} \mathrm{He}$ probes. Due to the scarcity of ${ }^{3} \mathrm{He}$, in this work, we look for an alternative based on Li-Glass scintillators.

Using SiPM light sensors instead of PMTs, in the same detector dimensions, the volume of the moderator and the reflector can be increased, thus increasing the sensitivity. To achieve an optimal configuration, we performed MCNP simulations. Among the best results, it is worth mentioning a detector that includes a $24 \mathrm{~cm} \times 19 \mathrm{~cm} \times 9 \mathrm{~cm}$ polypropylene moderator. Behind the $4 \mathrm{~cm}$ moderator layer, there is a $200 \mathrm{~mm} \times 150 \mathrm{~mm}, 1 \mathrm{~mm}$ thick Li-Glass scintillator. Arrays of SiPMs attached to one face of the scintillator, collect the output light producing a measurable voltage pulse. The described dimensions include also a 2 $\mathrm{cm}$ reflector layer on each side of the scintillator and a layer of $4 \mathrm{~cm}$ polypropylene behind the SiPMs. MCNP simulations show a sensitivity of approximately $46 \mathrm{cps} / \mathrm{nv}$. Although in practice, in approximately $80 \%$ of the neutron interactions, the output pulse has sufficient amplitude to register as a neutron pulse, the described configuration and simulation results show a good alternative to meet IEC 62534 sensing performances instead of previous solutions based on ${ }^{3} \mathrm{He}$ tubes.

Measurement Results: The performances of the developed probe were evaluated with a $13000 \mathrm{n} / \mathrm{s}{ }^{252} \mathrm{Cf}$ source. The source, located $30 \mathrm{~cm}$ in front of the detector, produced a flow of $1.15 \mathrm{n} / \mathrm{s}$; maintaining the same measurement conditions we examined scintillators of 1 $\mathrm{mm}$ and $2 \mathrm{~mm}$ thickness. Measurement results show very low differences in their efficiencies: $2.4 \mathrm{cps} / \mathrm{nv}$ compared 
to $2.5 \mathrm{cps} / \mathrm{nv}$ for the $1 \mathrm{~mm}$ and $2 \mathrm{~mm}$ thick scintillators, respectively.

One of the most important parameters in the performance of a neutron search detector is its immunity to the gamma radiation. In Li-Glass scintillators, the gamma equivalent energy of the neutron photo-peak is about 1.6 $\mathrm{MeV}$. Since the measured full width of this photo-peak is greater than $30 \%$, there is the possibility that high-energy gamma rays are incorrectly measured as neutrons. it should be noted that in high-intensity gamma fields, there is pulse pile-up. Hence, some of these pulses are erroneously registered as neutron pulses. Since the effect increases with the scintillator thicknesses, in many applications, it may be preferable to use a relatively thin, $1 \mathrm{~mm}$ thick scintillator to improve the gamma radiation immunity and increase the volume of the moderator to improve the neutron sensitivity.

\section{CONCLUSIONS}

Models of neutron detectors consisting of Li-Glass scintillators coupled to arrays of SiPMs and appropriate moderator configuration are presented. Measurements performed with these models show that Li-Glass scintillators coupled to arrays of SiPMs are an effective alternative to the PMT light sensors while maintaining and even improving the detection efficiency and reducing dimensions, weight and energy consumption. Neutron sensitivity measurements with a $1 \mathrm{~mm}$ thick scintillator were $10 \%$ lower than with a $2 \mathrm{~mm}$ thick scintillator, but the gamma rejection is better than twice that with the thin scintillator, which greatly improves the compatibility of the presented technology with the IEC 62534 standard requirements. According to measurements made with the presented neutron sensors and the results of MCNP simulations for a larger detector, using the described technology, it is possible to develop detectors that comply with the dimension and weight allowed by the IEC 62534 standard for these types of instruments, and as well, exceeding the efficiency requirements for neutron detection.

\section{ACKNOWLEDGMENT}

We would like to thank Rotem Industries Ltd., for their help in assembling the detectors and conducting experiments with their equipment and installations.

\section{REFERENCES}

[1] R. C. Runkle, A. Bernstein, and P. E. Vanier, "Securing special nuclear material: Recent advances in neutron detection and their role in nonproliferation," J. Appl. Phys., vol. 108, no. 11, 2010, Art. no. 111101, doi: 10.1063/1.3503495.

[2] R.T. Kouzes et al., "Neutron detection alternatives to ${ }^{3} \mathrm{He}$ for national security applications", Nucl. Instrum. Methods Phys. Res. A vol. 623, pp. 1035-1045, 2010, doi: 10.1016/j.nima.2010.08.021.
[3] "Introduction to SiPM Technical Note, Rev 6.0," SensL, Feb. 2017. Accessed: Mar. 2019. [Online]. Available: tps://www.sensl.com/downloads/ds/TN\%20\%20Intro\%20to\%20SPM\%20Tech.pdf

[4] Radiation Protection Instrumentation — Highly Sensitive Hand-held Instruments for Neutron Detection of Radioactive Material, IEC-62534, International Electrotechnical Commission, Geneva, June 2010.

[5] "6-Lithium glass bespoke to your application," Scintacor, Accessed: Mar. 14, 2019. [Online]. Available: https://scintacor.com/products/6-lithiumglass/

[6] J.F. Briesmeister, "MCNP - A Monte Carlo NParticle Transport Code," Los Alamos Nat. Lab., NM, USA, , LA-13709-M, version 4C2, 2001. 